\title{
Symptomatic knee osteoarthritis and dyslipidaemia
}

\author{
Authors: Asso Amin, ${ }^{A}$ Raof Merza, ${ }^{A}$ Mohammed ] Baban, ${ }^{B}$ Hawar Khan, ${ }^{B}$ Khalid A Hama-ghareeb, ${ }^{A}$ \\ Mohammed IM Gubari, ${ }^{A}$ Soran Noori, ${ }^{C}$ Saman Sadeq ${ }^{C}$ and Alan Saeed ${ }^{D}$
}

\section{Background}

As a complex multifactorial condition, knee osteoarthritis has been considered as a leading cause of disability. Dyslipidaemia is a metabolic component that can probably play a role in knee osteoarthritis development and comorbidities; however, this relationship is still debated. This study was carried out in order to figure out the prevalence of dyslipidaemia among knee osteoarthritis patients and compare their abnormal serum lipid components with non-exposed individuals.

\section{Patients and methods}

A total of 60 patients with knee osteoarthritis and 60 non-exposed (without knee osteoarthritis) individuals were studied in a prospective cohort study that was conducted from March 2018 to May 2019. The patients were chosen from those who were referred to the Rheumatology Division in Sulaymaniyah, the Kurdistan region of Iraq. European League Against Rheumatism and American College of Rheumatology diagnostic and classification criteria and radiographic confirmation for definite osteophytes were utilised to diagnose the primary knee osteoarthritis. Required data were collected using a questionnaire, taking blood samples and conducting several laboratory tests.

\section{Results}

The mean age of the patients with knee osteoarthritis was 51.8 years. Female-to-male ratio was 2.1:1. It was seen that dyslipidaemia increased twofold among patients with knee osteoarthritis compared with the non-exposed individuals. Patients and non-exposed individuals were significantly different in terms of dyslipidaemia prevalence $(\mathrm{p}<0.013)$. Furthermore, all the lipid components were significantly abnormal in those with knee osteoarthritis.

\section{Conclusion}

Dyslipidaemia is prevalent among knee osteoarthritis patients, and there is a significant association between knee

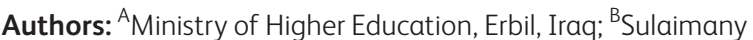
General Medical Teaching Hospital, Sulaimany, Iraq; 'Sulaimany Surgical Teaching Hospital, Sulaimany, Iraq; ${ }^{D}$ Primary Health Care Corporation, Doha, Qatar osteoarthritis and high-density lipoprotein, total cholesterol, low-density lipoprotein and triglyceride. Dyslipidaemia prevention may reduce the development of knee osteoarthritis and cardiovascular comorbidities.

\section{Conflicts of interest}

None declared. 